\title{
Andreas Vogel
}

Am 17. November 2004 wurde Dr. Andreas Vogel, Köln, durch die Fakultät »Sprach- und Literaturwissenschaften« der Otto-Friedrich-Universität Bamberg die Venia legendi für das Fach Kommunikationswissenschaft verliehen. Der wissenschaftliche Vortrag behandelte das Thema "Das Internet - ein Medium? Der Medienbegriff und die Online-Welt«, die öffentliche Vorlesung trug den Titel »Die Erschließung ausländischer Märkte: Strategien deutscher Medienkonzerne». Schriftliche Grundlage der kumulativen Habilitation waren die selbstständige Veröffentlichung "Die populäre Presse" (München 1998) und der Aufsatz »Pressegattungen im Zeitschriftengewand « im von Vogel zusammen mit Christina Holtz-Bacha herausgegebenen Band "Zeitschriften und Zeitschriftenforschung" (Sonderheft 3/2002 der $>$ Publizistik $`$.

Obwohl Vogel ein weit breiteres wissenschaftliches Euvre mit zahlreichen Aufsätzen in der Fachpresse vorweisen kann, stützte sich die Begutachtung seiner schriftlichen Leistungen bewusst auf das Minimum, das in einem kumulativen Verfahren vorgesehen ist. Damit konnte Vogel verdeutlichen, dass die Monographie eine habilitationswürdige Einzelleistung darstellt. Die schriftliche Habilitationsleistung wurde von Oliver Jahraus, Rudolf Stöber, Anna Maria Theis-Berglmair (Bamberg) und Jürgen Wilke (Mainz) begutachtet.

Andreas Vogel kommt das Verdienst zu, mit seiner Monografie die Zeitschriftenforschung in Deutschland aus einem Dornröschenschlaf geweckt zu haben. Die hohe Zeit der Zeitschriftenforschung reichte von den 1920er Jahren bis in die 1960er und 1970er Jahre mit den einschlägigen, allerdings zumeist historisch orientierten Standardwerken von Joachim Kirchner, Wilmont Haacke und Jürgen Wilke. Zeitschriftenforschung zu aktuellen Entwicklungen fand in der Kommunikationswissenschaft fast nicht mehr statt. Das ist um so bedauerlicher, als gerade seit Mitte der 1970er Jahre das Zeitschriftensegment sich zum dynamischsten Sektor der Medien entwickelte. Vogel hat mithin einen blinden Fleck der kommunikationswissenschaftlichen Forschung entdeckt. Seine Monografie zeichnet sich zunächst durch eine akribisch genaue Bestandsaufnahme der Entwicklungen im Zeitschriftenmarkt zwischen 1975 und 1995 aus. Mit seinem induktiven Vorgehen entwickelt Vogel ein schlüssiges Kategorienschema der Zeitschriftenpresse, das die Wissenschaft in diesem Gebiet erstmalig von den Vorgaben der kommerziellen Statistik unabhängig gemacht hat. Darüber hinaus liegt der Wert seiner Forschung in der Aufdeckung unterschwelliger Innovationsmuster. Vogel hat mit seiner Arbeit die Zeitschriften als zeitgenössische Quelle des sozialen Wandels neu entdeckt.

Andreas Vogel wurde am 12.9.1960 in Magdeburg geboren. Nach Studium (1980-1987) und Promotion (1989) in Münster arbeitete er von 1990 bis 1996 in Lüneburg als Wissenschaftlicher Assistent an der dortigen Universität. Seit 1996 ist er Miteigentümer und Geschäftsführer des »Wissenschaftlichen Instituts für Presseforschung und Medienberatung« in Köln. 